Article

\title{
Translation, Cultural Adaptation of Spiritual Needs Questionnaire in Pakistan
}

\author{
Aisha Kashif ${ }^{1, *}$ and Zaira Kanwal ${ }^{2}$ \\ 1 Faculty of Humanitites, COMSATS University, Park Road, Chak Shehzad, Islamabad 45550, Pakistan \\ 2 Department of Humanities, COMSATS University, Park Road, Chak Shehzad, Islamabad 45550, Pakistan; \\ zaira_kanwal@yahoo.com \\ * Correspondence: aisha.kashif@comsats.edu.pk
}

Received: 1 March 2018; Accepted: 3 May 2018; Published: 18 May 2018

\begin{abstract}
The current study was conducted with the aim of translating, adapting, and exploring the factor structure of Spiritual Needs Questionnaire (SpNQ) in chronically ill patients. To meet this objective, the English-version SpNQ was translated into Urdu for Pakistan following standard methods of translation and adaptation. The Urdu version was then used to collect data from a sample of 150 chronically ill patients. The results showed that spiritual needs were significantly associated with each other. Compared to the previous English-version that proposed a four-factor solution, the exploratory factor analysis revealed a three-factor structure for the Urdu version with good internal consistency coefficients, indicating the new version to be a reliable measure.
\end{abstract}

Keywords: spiritual needs; translation; exploratory factor analysis; internal consistency

\section{Introduction}

Spirituality is a multifaceted term that is represented diversely in practice and perception. Spirituality originated from the Latin word Spiritus, which means to breathe, and while spirit refers to purity of soul. The concept of spirituality emerged from Christianity, denoting a life oriented toward the Holy Spirit (Mok et al. 2010). Some authors suggest spirituality to be a form of religious transformation, a process of redeeming originality of an individual directed toward an image of God in terms of divinity and self-actualization (Saucier and Skrzypińska 2006). For achieving this transformation in every belief system, there are different codes of submission. For example, there is the Torah in Judaism, Christ in Christianity, the Buddha in Buddhism, and the Prophet Muhammad (peace be upon him) in Islam (McMahan 2008).

According to (Ellison 2006), spirituality is a motivational force or power that leads an individual towards path of curiosity, surge, purpose, direction and meaning in life. It is the totality of human existence and does not exist independent of mind and body (psyche, soma). Spirituality can also be defined as way individuals experience their relatedness with their family, self, others and the transcendent (Puchalski et al. 2009). Spirituality is a common human attribute found in all individuals whether they are religious or not (Woll et al. 2008). Koenig et al. (2012) maintained that spirituality is sanctity within as well as at the extrinsic level. In the Islamic context, sufism is considered a form of spirituality in which a spiritual leader or peer transmits spiritual knowledge to his students. Tasawwuf or Sufiism is a mystical aspect of Islam (Azeemi 2005).

Spirituality has been indicated as a significant factor in wellbeing and quality of life amongst the patient population, especially those diagnosed with cancer (Balboni et al. 2007). The quality of life for patients constitutes psychosocial, physical, and spiritual wellbeing that helps individuals to grow in totality (Oh and Kang 2005). Spiritual wellbeing is an overall state that fosters positive growth and survival that is responsible for an increased sense of connectivity with a divine power 
(Shahbaz and Shahbaz 2015). Literature suggests a positive correlation between spiritual wellbeing and psychological wellbeing (Moreira-Almeida et al. 2006).

The existence of individuals as humans depends on the relation between biological, psychological, spiritual, and social dimensions. The biopsychosocial-spiritual model supplements holistic grounds for treating and healing individuals and also provides a conceptual basis for addressing overall health and care (Sulmasy 2002). Spiritual needs exist in both the clinical and non-clinical population. Spirituality serves as a significant factor in making a contribution toward physical health, most prominently in cancer patients. It also escalates their self-worth, emotional support, coping with illness, and hope (Thune-Boyle et al. 2006). A relatively recent study (Büssing et al. 2010) assessed the spiritual, psychosocial, and existential needs of patients with chronic pain disease.

The study investigated spirituality/religiosity as significant source of healing and coping amongst European patients with chronic illnesses (Büssing et al. 2010). In the following study, the psychosocial needs, spiritual, and existential needs of patients were assessed among those chronically ill patients who did not consider themselves to be religious (Büssing et al. 2009, 2007a, 2007b). The results of this study showed that need for inner peace and actively giving were significantly high, whereas religious needs were of least importance among European cancer patients. It was additionally found that there is an association between spirituality and religiosity and coping mechanism against disease (Büssing et al. 2007a). In cancer patients particularly, spirituality serves to provide emotional support, hope, and meaning in life and therefore helped them to better cope with their illness. Another study revealed that cancer patients have a relatively high level of unmet spiritual needs in comparison to biological and psychosocial needs (Rainbird et al. 2009). These needs are associated with improved life quality and hope in life. The cancer patients in which these needs are less able to cope with their illness and have a reduced quality of life (Zamanzadeh et al. 2014).

The major objective of the present research is to translate and adapt the spiritual needs questionnaire (SpNQ). The instrument was developed by Büssing et al. (2010) considering patient population as spirituality and religion were perceived as an imperative technique of coping. Previous attempts concentrated on spiritual needs of ill, close to death while SpNQ was administered to chronically ill patients. SpNQ has been studied in different countries, including Malaysia, Nigeria, Poland (Büssing et al. 2015), France, China (Büssing et al. 2013), Germany (Büssing et al. 2018), England, and Iran (Moeini et al. 2018). The instrument has been normalized in Iran with negligible revisions. In gauging spiritual needs, it is important for the respondents' inner self to be revealed (Seddigh et al. 2016). The spiritual needs are stated to be interrelated; thus, cautious administration of instrument is suggested.

Some concepts included in the spiritual needs questionnaires are culturally dependent. For example, in western cultures, music was added as a putative need in patient care. but in later research, it was deleted. In Islamic contexts, listening to or reading the Quran (holy book) holds spiritual significance. Likewise, contingent upon the culture where the questionnaire is being used, the importance of needs will vary. For example, in the Chinese version, the need for active giving was found to be most important compared to the need for inner peace that emerged in German norms. Previous literature highlights a need for translation of the spiritual needs questionnaires as they are culturally dependent, according to different cultures with adjustment of certain items conveying culture-specific meaning. Considering the above commentary, the current research endeavored to translate, adapt, and validate the SpNQ in a Pakistani sample of chronically ill patients.

\section{Method}

\subsection{Measure}

Spiritual Needs Questionnaire. The original version of spiritual needs questionnaire (SpNQ) was developed by Büssing et al. (2010) to assess spiritual needs of patients with chronic illness. SpNQ is a 29-item tool with four subscales i.e., religious needs, existential needs, need for inner peace, and 
need for actively giving or generativity. SpNQ is rated on a four-point Likert scale ranging from $0=$ not at all, $1=$ somewhat $2=$ very, and $3=$ extremely. The Cronbach alpha for religious needs $=0.92$, existential needs $=0.82$, need for inner peace $=0.82$, need for active giving and generativity $=0.74$. The spiritual needs questionnaire is free from religious bias and is suitable to use both in secular states as well as religious states. The current study translated the original version into Urdu according to the following steps after obtaining permissions from the original author.

\section{Phase 1: Tool Translation and Adaptation of English Version of the Spiritual Needs Questionnaire}

The translation and adaptation process were completed in two phases. In the first phase, translation and adaptation of SpNQ was accomplished, and in second phase, the exploration of factor structure and validity of tool was established. All steps in tool translation were considered.

Step 1: Forward translation. Four translators from the Army Public School and College were approached who were bilingual and had postgraduate academic qualifications. The translators were requested to translate the original English tool into Urdu so that the inherent meaning of the items was unchanged and explicit i.e., ensure a parallel value of item content in both versions without any adaptation. After the translation, the translated versions were subjected to the committee approach step.

Step 2: Evaluation of translated items by a committee of experts. The objective of step 2 was to determine the unqualified items. In order to accomplish this, all translated statements from four translations were written down under the corresponding item. These items were then evaluated by members of a committee. The committee members included two Ph.D. professors-one from Quaid-e-Azam University and the other from COMSATS Institute of Information Technology Islamabad. Both were bilingual. All items were carefully assessed, and items having the most appropriate correspondence with original items in semantic terms were selected. Subsequently, items conveying approximately exact meaning of original English-version were chosen for back translation.

Step 3: Back Translation of the selected items into English. In order to corroborate equivalence of translated items, items of the Urdu version were translated back into English. For back translation, the translated version was given to five bilinguals who were not involved in the prior translation. The back translators were Ph.D. professors from Riphah University and Government College. Five translations were later given to committee for evaluation.

Step 4: Evaluation of back-translated items in to English. The back-translated items were written down under their corresponding original items for comparison. The committee constituted same members who were involved in back translation. No incomprehensibility was reported in back translated items. The comparison between back translated and original items to affirmed accuracy of translated version.

Step 5: Cultural equivalence. The Urdu version of the SpNQ was administered to patients with various chronic illnesses for at least three months. The main focus of this step was evaluating the comprehension of items and format. It was observed that, instead of self-administration, it was best to use it in a personal interview format.

The only change relative to the original was the format of administration of the questionnaire. The original English version was designed as self-applicable, whereas for the Brazilian-Portuguese (de Araujo Toloi et al. 2016) version, reading the questionnaire out loud to the patient during a personal interview due to the rate of illiteracy among patients was conducted. Thus, the phrase "when you read these statements" was changed to "when you listen to these statements," followed by the instruction to answer yes or no. The statements could be understood and answered by at least $95 \%$ of the patients, thus proving to be easy to understand, and no objections of cultural inappropriateness was reported.

\section{Phase 2: Exploration of Factor Structure for SpNQ Urdu Version}


The objectives of phase 2 included exploration of factor structure and psychometric examination for the newly translated Urdu version. To meet the phase 2 objectives, the newly translated Urdu version was administered to a local sample of chronically ill patients.

\subsection{Sample and Procedure}

The sample consisted of 150 in- and out-patients with chronic illnesses, having pathological conditions prevailing for three or more months along with persistent or recurring illness as diagnostic criteria. The patients included had illnesses histories of up to 12 years and above and under medical supervision with periodic visit to their physicians. The age ranged between 17 to 75 . The demographic properties included gender, marital status, chronic illness, and duration of chronic illness. The objective of including these variables was to observe gender differences concerning spiritual needs keeping in view previous findings. There were 46 males and 104 females. Marital status association with respect to spiritual needs in chronically ill patients has not previously explored, which present study intended to explore. There were no educational criteria for the sample population. The chronic illnesses included were classified in order of their chronicity, intensity, and frequency. The duration of the chronic illness has been found to be associated with increased spiritual concerns. To examine its impact, this variable duration was chosen. The sample was selected from hospitals in Islamabad and Rawalpindi using a purposive or selective sampling technique. The present sample and participants were interviewed with the translated Urdu version to gain insight along with accuracy of their responses. The same procedure was used for those patients who were confined to bed. Prior to the administration of the questionnaire, the patients were given instructions in simple language, and their consent was obtained. The codes of ethics for research were strictly followed, and typically, each participant took 15-20 min for a complete administration.

\section{Results}

The main aim of the present study was to translate, adapt, and explore the factor structure of the translated Urdu version of the Spiritual Needs Questionnaire. To meet the above objective, exploratory factor analysis (EFA) was conducted after considering the assumptions of EFA e.g., interval level and normality. The sample size was checked for adequacy of sample size, indicated by a Kaiser-Meyer-Olkin score greater than $0.5(\mathrm{KMO}=0.78, p<0.001)$. The communality pattern matrix in Table 1 explains the variance explained by each item. Principle component analysis was used to extract the maximum number of positive eigen-values in determining the dimensions without losing any information. It was observed that the maximum amount of variance was explained in the first two factors. According to indications that showed spiritual needs to be correlated, an oblique rotation with varimax strategy was employed. The results display that some items were cross-loaded when compared with the original English version. The cross-loading items were handled by retaining items in the factors where highest loadings were observed and loadings greater than 0.4 (Costello and Osborne 2005). It was observed that approximately $30 \%$ of the variance was explained by the two factors, a third factor explain 5\%, and the rest explaining 3\% of the variance. The scree plot also indicated a bent after the third factor, which was suggestive of a prominent role of the initial three-factor structure.

The EFA was rerun was after being restricted to a three-factor solution based on the information received from eigen-values (greater than 1), and scree plot, rejecting factors with fewer than three-items. After the re-execution of EFA, the first factor explained about $28 \%$ of the variance, with the second explaining $16 \%$, and the third factor explaining $12 \%$ of the variance with $52 \%$ of cumulative explained variance. 
Table 1. Communalities pattern matrix.

\begin{tabular}{lc}
\hline & Extraction \\
\hline To talk with others about your fears and worries? & 0.38 \\
That someone of your religious community (i.e., Alim, peer, imam.guru, padri) cares for you? & 0.05 \\
To reflect your previous life? & 0.52 \\
To dissolve open aspects of your life? & 0.28 \\
To plunge into beauty of nature? & 0.30 \\
To dwell at a place of quietness and peace? & 0.19 \\
To find inner peace? & 0.43 \\
To find meaning in illness and/or suffering? & 0.32 \\
To talk with someone about the question of meaning in life? & 0.33 \\
To talk with someone about the possibility of life after death? & 0.52 \\
To turn to someone in a loving attitude? & 0.28 \\
To give away something from yourself? & 0.36 \\
To solace someone? & 0.27 \\
To forgive someone from a distinct period of your life? & 0.53 \\
\hline
\end{tabular}

The rotated component matrix with fixed 3-factors solution (see Table 2) represented a balanced structure with eigen-values ranging between 0.41 to 0.72 . Further, the new factor structure showed that items in the inner peace need subscale in the original English version loaded onto the family social support needs after factor rotation. The final 15-item SpNQ questionnaire is available in an Urdu version that constitutes the spirituality needs within religious, family social support and existential needs. The number of items in each factor were 10,6 , and 7 , respectively.

Table 2. Rotated component matrix for the three-factor solution with oblique $(N=150)$.

\begin{tabular}{|c|c|c|c|c|c|}
\hline \multirow[t]{2}{*}{ Items } & \multicolumn{3}{|c|}{ Factors } & \multirow[b]{2}{*}{$M$} & \multirow[b]{2}{*}{$S D$} \\
\hline & 1 & 2 & 3 & & \\
\hline That someone prays for you? & 0.73 & & & 22.21 & 5.64 \\
\hline To pray with someone? & 0.72 & & & 22.14 & 5.62 \\
\hline To turn to a higher presence (i.e., God, Allah, Angels) & 0.72 & & & 22.14 & 5.62 \\
\hline To pray for yourself? & 0.67 & & & 19.61 & 5.44 \\
\hline To be forgiven? & 0.62 & & & 18.92 & 5.35 \\
\hline To forgive someone from a distinct period of your life? & 0.60 & & & 17.65 & 5.31 \\
\hline To find inner peace? & 0.58 & & & 16.94 & 4.97 \\
\hline To read religious/spiritual books? & 0.51 & & & 14.95 & 4.68 \\
\hline To participate at a religious ceremony & 0.43 & & & 13.98 & 3.47 \\
\hline To dwell at a place of quietness and peace? & 0.41 & & & 12.97 & 3.41 \\
\hline \multicolumn{6}{|l|}{$\begin{array}{l}\text { That someone of your religious community (i.e., Alim, peer, } \\
\text { imam.guru, padri) cares for you? }\end{array}$} \\
\hline For being complete and safe? & & 0.66 & & 17.92 & 4.35 \\
\hline To feel connected with family? & & 0.65 & & 17.65 & 4.31 \\
\hline To receive more support from your family? & & 0.64 & & 17.61 & 3.97 \\
\hline To pass own life experiences to others? & & 0.63 & & 15.95 & 3.68 \\
\hline To be re-involved by your family in their life concerns? & & 0.62 & & 15.78 & 3.47 \\
\hline To be assured that your life was meaningful and of value? & & 0.59 & & 13.97 & 3.41 \\
\hline To talk with someone about the possibility of life after death? & & & 0.70 & 18.72 & 5.35 \\
\hline To reflect your previous life? & & & 0.68 & 17.55 & 5.31 \\
\hline To give away something from yourself? & & & 0.59 & 15.94 & 4.97 \\
\hline To talk with others about your fears and worries? & & & 0.59 & 15.94 & 4.97 \\
\hline To talk with someone about the question of meaning in life? & & & 0.54 & 14.98 & 3.67 \\
\hline To find meaning in illness and/or suffering? & & & 0.49 & 13.97 & 3.41 \\
\hline To plunge into beauty of nature? & & & 0.45 & 11.92 & 2.35 \\
\hline \multicolumn{6}{|l|}{ To dissolve open aspects of your life? } \\
\hline \multicolumn{6}{|l|}{ To solace someone? } \\
\hline To turn to someone in a loving attitude? & & & & & \\
\hline
\end{tabular}

The new factor structure for the Urdu version was evaluated for internal consistency though Cronbach alpha. The results showed psychometrically sound values presented in the Table 3 . The correlation analysis revealed all subscales to be positively significantly correlated with each other, suggesting similar direction of elevation of decline for either. 
Table 3. Correlation coefficients for the Urdu version subscales $(N=150)$.

\begin{tabular}{llccccccc}
\hline & Factors & $\mathbf{1}$ & $\mathbf{2}$ & $\mathbf{3}$ & Items & $\boldsymbol{M}$ & $\boldsymbol{S D}$ & $\boldsymbol{\alpha}$ \\
\hline 1 & Religious Needs & - & $0.46^{* *}$ & $0.26^{* *}$ & 10 & 24.13 & 5.78 & 0.82 \\
2 & Family Social & & - & $0.19^{*}$ & 7 & 11.35 & 5.04 & 0.70 \\
3 & Support Needs & & & - & 6 & 12.66 & 4.41 & 0.75 \\
\hline
\end{tabular}

\section{Discussion}

The Spiritual Needs Questionnaire was designed to measure spirituality in patients, especially those suffering from chronic illnesses where the duration of illness is long-term and the illness is enduring in nature. In such conditions, the psychological and emotional wellbeing of patients is especially at stake. Spiritual coping is one of the ways the patients combat the challenges of chronic illnesses. Spirituality is a varied concept that is indicated previously to be embedded within religious, cultural, and ethnic practices. This is especially in Pakistan, where the spiritual practices follow religious teachings as well as an influence of Indo-Pak historical influences. Nevertheless, the spiritual needs emerge in the lifespan of every individual and may surface early when faced with life-threatening challenges.

Presently, the current study contributed in broadening the scope of existing Spiritual Needs Questionnaire by translating, adapting, and establishing psychometrics for the tool. Büssing et al. (2010) suggested that when using Spiritual Needs Questionnaire, it must consider that these needs may follow different patterns or dimensions e.g., in the Iranian version, a four-factor structure emerged. Similarly, in the Portuguese version, a five-factor structure differentiated inner peace, religious, existential, actively giving/generativity needs, and family social support needs. The Chinese and Polish versions hold similar factor structures (Büssing et al. 2018). Therefore, the need for further exploration of factor structure in different contexts is highlighted. The current findings presented a diverse picture of the construct compared to the original version. Previously conducted research has identified four dimensions of needs in patients with any chronic or terminal illness. These needs include religious needs, existential needs, inner peace needs, and giving or generativity needs. The factor structure of spiritual needs in Pakistan is different as compared to given structures prevailing in western states. In Pakistan, these four needs cluster in three factors, into which all other needs are accommodated. These needs include religious needs, family social support needs, and existential needs. The inner peace need items were found to merge with family social support needs. The family social support needs were not primarily intended to be spiritual in nature. After exploratory and confirmatory factor analysis, it was found that the spiritual need items merged with family social support needs.

The collectivistic context of present study provides a logical explanation for this merger. Commonly, family plays a pertinent role in an experience of illness and also provides spiritual or religious support, particularly coming from the elderly members of the family. A common practice reflecting such behaviors is asking elderly members of family to conduct praying rituals for the ill. The family also serves as spiritual mentors especially in the lives of chronically ill patients. Family members create an active collaboration with patient to improve their socialization practices during course of illness (Rosland and Piette 2010). Family members also provide spiritual care to patients, thereby helping them in acceptance of disease and to develop the potential for painful treatment procedures. Therefore, family social support needs are surfaced as spiritual items within the context of Pakistan.

Religious needs include being involved in religious services to form a transcendent connection with God. In the context of Pakistan, religious needs occupy a prominent locus in people's lives because of strong religious and spiritual beliefs. An inclination toward religion and spirituality after diagnosis is used as mechanism to adapt disease conditions (Lim 2013) in that family social support 
works as a moderator, palliating them to fulfill their religious needs. Although this can further be attributed to the larger portion of the sample being married, the unmarried participants also relied on family members for meeting their spiritual needs.

The existential needs include discussing life after death, solutiosn for apparent problems, reflecting on one's previous life, and discussing the meaning in life. The majority of chronically ill patients reported that soliciting contiguity to Allah is a very powerful way to cope with disease. Many patients responded that they are not afraid of disease because the illness comes from God and he will alleviate it. This was consistent with the findings of (Nixon et al. 2013). They also believed that this illness might be their trial in this world for which there will be a reward in the afterlife.

This translated version of the spiritual need questionnaire measures the spiritual need of only chronically ill patients. The scope should be further broadened to assess the spiritual needs of those people who are not chronically ill.

Author Contributions: Conceptualization, A.K. and Z.K.; Methodology, A.K.; Software, SPSS (version 22); Validation, A.K. and Z.K.; Formal Analysis, A.K.; Investigation, A.K. and Z.K.; Resources, A.K. and Z.K.; Data Curation, Z.K.; Writing-Original Draft Preparation, Z.K.; Writing-Review \& Editing, A.K.; Visualization, A.K. and Z.K.; Supervision, A.K.; Project Administration, A.K.

Funding: This research received no external funding.

Conflicts of Interest: The authors declare no conflict of interest.

\section{References}

Azeemi, Khwaja Shamsuddin. 2005. Muraqaba: The Art and Science of Sufi Meditation. Houston: Plato Publishing Inc. Balboni, Tracy A., Lauren C. Vanderwerker, Susan D. Block, M. Elizabeth Paulk, Christopher S. Lathan, John R. Peteet, and Holly G. Prigerson. 2007. Religiousness and spiritual support among advanced cancer patients and associations with end-of-life treatment preferences and quality of life. Journal of Clinical Oncology 25: 555-60. [CrossRef] [PubMed]

Büssing, Arndt, Thomas Ostermann, and Peter F. Matthiessen. 2007a. Adaptive coping and spirituality as a resource in cancer patients. Breast Care 2: 195-202. [CrossRef]

Büssing, Arndt, Thomas Ostermann, and Harold G. Koenig. 2007b. Relevance of religion and spirituality in German patients with chronic diseases. The International Journal of Psychiatry in Medicine 37: 39-57. [CrossRef] [PubMed]

Büssing, Arndt, Andreas Michalsen, Hans-Joachim Balzat, Ralf-Achim Grünther, Thomas Ostermann, Edmund A. M. Neugebauer, and Peter F. Matthiessen. 2009. Are spirituality and religiosity resources for patients with chronic pain conditions? Pain Medicine 10: 327-39. [CrossRef] [PubMed]

Büssing, Arndt, Hans-Joachim Balzat, and Peter Heusser. 2010. Spiritual needs of patients with chronic pain diseases and cancer-validation of the spiritual needs questionnaire. European Journal of Medical Research 15: 266. [CrossRef] [PubMed]

Büssing, Arndt, Xiao-Feng Zhai, Wen-Bo Peng, and Chang-Quan Ling. 2013. Psychosocial and spiritual needs of patients with chronic diseases: Validation of the Chinese version of the Spiritual Needs Questionnaire. Journal of Integrative Medicine 11: 106-15. [CrossRef] [PubMed]

Büssing, Arndt, Iwona Pilchowska, and Janusz Surzykiewicz. 2015. Spiritual needs of Polish patients with chronic diseases. Journal of Religion and Health 54: 1524-42. [CrossRef] [PubMed]

Büssing, Arndt, Daniela Rodrigues Recchia, Harold Koenig, Klaus Baumann, and Eckhard Frick. 2018. Factor Structure of the Spiritual Needs Questionnaire (SpNQ) in Persons with Chronic Diseases, Elderly and Healthy Individuals. Religions 9: 13. [CrossRef]

Costello, Anna B., and Jason W. Osborne. 2005. Best practices in exploratory factor analysis: Four recommendations for getting the most from your analysis. Practical Assessment, Research E Evaluation 10: 1-9.

de Araujo Toloi, Diego, Deise Uema, Felipe Matsushita, Paulo Antonio da Silva Andrade, Tiago Pugliese Branco, Fabiana Tomie Becker de Carvalho Chino, and Daniel P. Sulmasy. 2016. Validation of questionnaire on the Spiritual Needs Assessment for Patients (SNAP) questionnaire in Brazilian Portuguese. Ecancermedicalscience, 10. [CrossRef] [PubMed] 
Ellison, Lori L. 2006. The Spiritual Well-Being Scale. NewsNotes. 44. Available online: http:/ / mds.marshall.edu/ Co_faculty/9/ (accessed on 16 July 2006).

Koenig, Harold George, Dana King, and Verna B. Carson. 2012. Handbook of Religion and Health. New York: Oup Usa.

Lim, Kokkwang. 2013. Clinical hypnosis in the management of panic disorder with cognitive-behavioural and spiritual strategies. Australian Journal of Clinical E Experimental Hypnosis 41: 213-24.

McMahan, David L. 2008. The Making of Buddhist Modernism. Oxford: Oxford University Press.

Mok, Esther, Frances Wong, and Daniel Wong. 2010. The meaning of spirituality and spiritual care among the Hong Kong Chinese terminally ill. Journal of Advanced Nursing 66: 360-70. [CrossRef] [PubMed]

Moreira-Almeida, Alexander, Francisco Lotufo Neto, and Harold G. Koenig. 2006. Religiousness and mental health: A review. Revista Brasileira de Psiquiatria 28: 242-50. [CrossRef] [PubMed]

Moeini, Babak, Hadi Zamanian, Zahra Taheri-Kharameh, Tahereh Ramezani, Mohamadhasan Saati-Asr, Mohamadhasan Hajrahimian, and Mohammadali Amini-Tehrani. 2018. Translation and Psychometric Testing of the Persian Version of the Spiritual Needs Questionnaire among elders with chronic diseases. Journal of Pain and Symptom Management 55: 94-100. [CrossRef] [PubMed]

Nixon, Aline Victoria, Aru Narayanasamy, and Vivian Penny. 2013. An investigation into the spiritual needs of neuro-oncology patients from a nurse perspective. BMC Nursing 12: 2. [CrossRef] [PubMed]

Oh, Pok Ja, and Kyung Ah Kang. 2005. Spirituality: Concept analysis based on hybrid model. Journal of Korean Academy of Nursing 35: 709-20. [CrossRef] [PubMed]

Puchalski, Christina, Betty Ferrell, Rose Virani, Shirley Otis-Green, Pamela Baird, Janet Bull, Harvey Chochinov, George Handzo, Holly Nelson-Becker, Maryjo Prince-Paul, and et al. 2009. Improving the quality of spiritual care as a dimension of palliative care: The report of the Consensus Conference. Journal of Palliative Medicine 12: 885-904. [CrossRef] [PubMed]

Rainbird, Kathy, J. Perkins, Robert Sanson-Fisher, I. Rolfe, and Paul Anseline. 2009. The needs of patients with advanced, incurable cancer. British Journal of Cancer 101: 759. [CrossRef] [PubMed]

Rosland, Ann-Marie, and John D. Piette. 2010. Emerging models for mobilizing family support for chronic disease management: a structured review. Chronic Illness 6: 7-21. [CrossRef] [PubMed]

Saucier, Gerard, and Katarzyna Skrzypińska. 2006. Spiritual but not religious? Evidence for two independent dispositions. Journal of Personality 74: 1257-92. [CrossRef] [PubMed]

Seddigh, Ruohollah, Amir-Abbas Keshavarz-Akhlaghi, and Somayeh Azarnik. 2016. Questionnaires Measuring Patients' Spiritual Needs: A Narrative Literature Review. Iranian Journal of Psychiatry and Behavioral Sciences 10: e4011. [CrossRef] [PubMed]

Shahbaz, Kanwai, and Kiran Shahbaz. 2015. Relationship between Spiritual Well-being and Quality of Life among Chronically Ill Individuals. The International Journal of Indian Psychology 2: 128-42.

Sulmasy, Daniel P. 2002. A biopsychosocial-spiritual model for the care of patients at the end of life. The Gerontologist 42 S3: 24-33. [CrossRef]

Thune-Boyle, Ingela C., Jan A. Stygall, Mohammed R. Keshtgar, and Stanton P. Newman. 2006. Do religious/spiritual coping strategies affect illness adjustment in patients with cancer? A systematic review of the literature. Social Science \& Medicine 63: 151-64.

Woll, Monica L., Daniel B. Hinshaw, and Timothy M. Pawlik. 2008. Spirituality and religion in the care of surgical oncology patients with life-threatening or advanced illnesses. Annals of Surgical Oncology 15: 3048-57. [CrossRef] [PubMed]

Zamanzadeh, Vahid, Maryam Rassouli, Abbas Abbaszadeh, Alireza Nikanfar, Hamid Alavi-Majd, and Akram Ghahramanian. 2014. Factors influencing communication between the patients with cancer and their nurses in oncology wards. Indian Journal of Palliative Care 20: 12. [PubMed]

(C) 2018 by the authors. Licensee MDPI, Basel, Switzerland. This article is an open access article distributed under the terms and conditions of the Creative Commons Attribution (CC BY) license (http://creativecommons.org/licenses/by/4.0/). 